\title{
Control of Enterococcus faecalis Sex Pheromone cAD1 Elaboration: Effects of Culture Aeration and pAD1 Plasmid-Encoded Determinants
}

\author{
Keith E. Weaver* and Don B. Clewell ${ }^{\dagger}$ \\ *Department of Microbiology, School of Medicine, University of South Dakota, Vermillion, South Dakota 57069; \\ and $\nmid$ Departments of Oral Biology and Microbiology/Immunology, Schools of Dentistry and Medicine, \\ University of Michigan, Ann Arbor, Michigan 48109
}

Received November 28, 1990; revised March 4, 1991

\begin{abstract}
Aeration of plasmid-free Enterococcus faecalis strains resulted in an 8- to 16-fold decrease in sex pheromone CADI activity in culture filtrates. Levels of two unrelated pheromones, $\mathrm{CPD} 1$ and cAM373, were unaffected by culture aeration. Aeration also resulted in a decrease in the expression of conjugative transfer functions observed in cells containing pADI traB mutations, verifying a link between $\operatorname{tr} a B$ function and pheromone "shutdown." Tests with a series of pAD1 mini-plasmids indicated that the product of the $t r a B$ gene was involved in, but not sufficient for, pheromone shutdown; the cooperation of one or more other gene products encoded within the pheromone response control region was required. (c) 1991 Academic Press, Inc.
\end{abstract}

Plasmid-free strains of Enterococcus faecalis excrete a number of small peptide sex pheromones which induce a mating response in strains containing certain plasmids native to this organism. Each individual pheromone is specific for a different plasmid or family of plasmids. When plasmid-containing cells are exposed to the specific pheromone, they respond by synthesizing a proteinaceous "aggregation substance," localized to the cell surface, which facilitates the formation of mating aggregates; other functions required for transfer of plasmid DNA are also induced. Acquisition of the plasmid results in the loss of detectable pheromone activity specific for that plasmid. Pheromones specific for other plasmids continue to be elaborated. Because the precise mechanism, or mechanisms, by which a plasmid controls elaboration of its pheromone is unknown, the phenomenon has been called "pheromone shutdown" and this term is used throughout the text. Plasmid-containing cells also excrete another peptide, called inhibitor, which specifically and competitively inhibits the activity of the related pheromone. (For a recent review of pheromone-responsive conjugative systems, see Clewell and Weaver, 1989.)

The regulatory genes for the conjugative response of $E$. faecalis plasmid pAD1 to its specific pheromone, cAD1, are clustered in an approximately $7-\mathrm{kb}$ region of plasmid DNA (Ehrenfeld and Clewell, 1987; Ike and Clewell, 1984; Weaver and Clewell, 1988, 1989) (see Fig. 1). Transposon mutagenesis using $\operatorname{Tn} 917$ and Tn917-lac identified at least four genetic determinants essential for control of the pheromone response: (i) the $\mathrm{E}$ region, which is required for expression of both aggregation and transfer functions and is believed to include a positive regulatory determinant; (ii) the traA gene, which is required for the repression of the $\mathrm{E}$ region in the absence of CAD 1 and for signal transduction; (iii) the $\mathrm{C}$ region, which appears to be involved in signal sensing; and (iv) the $\operatorname{traB}$ gene. Because mutations in the $\operatorname{tr} a B$ gene resulted in derepression of aggregation and transfer functions without apparent production of endogenous CAD1, it was believed originally that the product of this gene was a negative regulator, perhaps functioning in co- 
operation with the product of the traA gene (Ike and Clewell, 1984). Recently, however, two plasmid derivatives, each with a lesion in the $\operatorname{tr} a B$ gene, which failed to properly shut down cAD1 production were isolated. One plasmid, pAM2030, contained a Tn917-lacderived deletion of a portion of $\operatorname{traB}$ and nearly all of the adjacent $C$ region (Clewell and Weaver, 1989). The other, pAM2045, contained two Tn917-lac inserts, one in $\operatorname{traB}$ and the other in the $E$ region (Weaver and Clewell, 1990). As the only common defect between these plasmids was in the $\operatorname{tr} B$ gene, these results suggested that the product of that gene is involved in pheromone shutdown. Extrapolating from these results, it was postulated that the $\operatorname{traB}$ phenotype, i.e., the constitutive expression of conjugative functions, was due to a response to endogenously produced CAD1 that was improperly regulated. However, this was difficult to prove since $\mathrm{pAD} 1$ derivatives containing $\operatorname{Tn} 917$ or $\operatorname{Tn} 917-$ lac single inserts within the traB region do not produce detectable cAD1. (It was observed, however, that strain-related variability in $\operatorname{tra} B$ phenotype correlated with the level of CAD1 produced by the host in the absence of pAD1.) Because a second mutation within either the pADI E or the C region was required to allow production of detectable $\mathrm{CAD} 1$, it was postulated that gene products encoded within these regions also played a role in pheromone shutdown.

In this study we report the identification of a single environmental factor, culture aeration, which decreased the activity of cAD1 and the expression of conjugative transfer functions of pADl traB mutants in parallel. Aeration affected the phenotype of single Tn917-lac insertions in traB even though no detectable cADl was produced in such strains. Results presented indicate that the traB phenotype results from a response to endogenously produced cAD1 and, therefore, that the $t r a B$ gene product is essential for cAD1 shutdown. Aeration has no effect on the phenotypes of $t r a A, \mathrm{C}$ region, or E region mutants, indicating that the products of these genes function in some aspect of the regula- tion of the pheromone response other than or in addition to pheromone shutdown. However, analysis of mini-plasmid constructs proved that $\operatorname{tr} a B$ was not sufficient for $\mathrm{CAD} 1$ shutdown, but required the function of an $E$ region or $\mathrm{C}$ region product(s), indicating that pheromone activity is controlled by multiple genes.

\section{MATERIALS AND METHODS}

Bacterial strains and plasmids. E. faecalis strain OG1X is a streptomycin-resistant, extracellular protease-deficient derivative of OG1S (Ike et al., 1983). The unrelated E. faecalis strain FA2-2 is a rifampin- and fusidic acid-resistant derivative of $\mathrm{JH} 2$ (Clewell $e t$ al., 1982). pAD1 derivatives used and constructed in this study are shown in Fig. 1 and their phenotypes described in Table 1.pPD1 is a 56-kb plasmid encoding bacteriocin and responding to the pheromone cPD1 (Dunny et al., 1978). pAM373 is a 36-kb cryptic plasmid responding to cAM373 (Clewell et al., 1985).

Media, growth conditions, and culture filtrate preparation. All cultures were grown in N2GT medium [nutrient broth No. 2 (Oxoid Ltd., London, UK) supplemented with $0.1 \mathrm{M}$ Tris buffer ( $\mathrm{pH} \mathrm{7.5)}$ and $0.2 \%$ glucose]. Solid medium was prepared by adding $1.8 \%$ agar (Difco Laboratories, Detroit, MI). Still cultures were grown in $5 \mathrm{ml}$ of medium in $20-\mathrm{ml}$ culture tubes at $37^{\circ} \mathrm{C}$. Aerated cultures were grown in $5 \mathrm{ml}$ of medium in a 125-ml Erlenmeyer flask shaken at $200 \mathrm{rpm}$ on a rotary shaker at $37^{\circ} \mathrm{C}$. Shaken anaerobic cultures were also grown in $5 \mathrm{ml}$ of medium in a 125 ml flask, but the flask was introduced into a Gas Pak (American Scientific Products, McGaw Park, IL) anaerobic jar which was then allowed to charge for $2 \mathrm{~h}$ at room temperature followed by shaking at $200 \mathrm{rpm}$ on a rotary shaker. For routine culture filtrate preparation from still and aerated cultures, cells grown to stationary phase in N2GT, or N2GT supplemented with $10 \mu \mathrm{g}$ of erythromycin $/ \mathrm{ml}$ in the case of plasmid-containing strains, were used to inoculate N2GT me- 


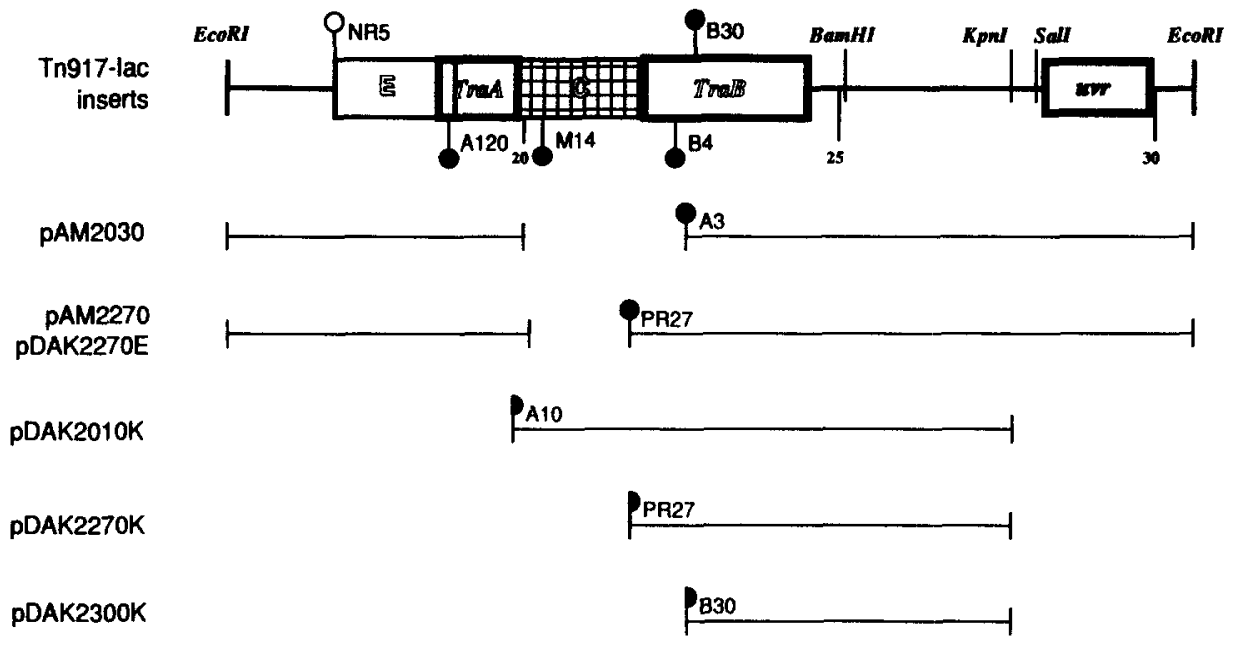

FIG. 1. Map of the pAD1 pheromone response regulatory region and relevant derivatives. Positions of Tn917-lac inserts are marked with circles or half-circles and stems, and the inserts are named according to previous convention. (Positioning of insert markers at top and bottom of map indicates opposite orientations.) Kilobase markers are shown below the reference maps and correspond to positions on the intact pAD1 plasmid. Plasmids pAM2120, pAM2140, pAM2040, and pAM2300 contain inserts A120, M14, B4, and B30, respectively, on intact pAD1, pAM2040E and pDAK2300E contain inserts B4 and B30, respectively, on a mini-plasmid consisting only of the $E c o$ RI fragment shown. pAM2005A contains the cADI-inducible Tn917-lac fusion NR5. pAM2125, pAM2145, and pAM2045 contain the NR5 fusion along with $\mathrm{A} 120, \mathrm{M} 14$, and B4, respectively. Lines below the reference map indicate the extent of deletions present in the plasmids noted along the left side. Plasmids pAM2030 and pAM2270 contain these deletions on an otherwise unmodified pAD1. pDAK2270E contains the same deletion as pAM2270 but consists only of the EcoRI fragment shown. pDAK2010K, pDAK2270K, and pDAK2300K are mini-plasmids consisting of only the portion of pADI indicated. The half-circles indicate deletion of $\mathrm{Tn} 917-$ lac beyond the nearly centrally located KpnI site. Phenotypes of these plasmids are summarized in Table 1.

dium at $2 \%(\mathrm{v} / \mathrm{v})$. Culture density was followed and cells were harvested in early stationary phase. Culture filtrates were prepared from supernatants as previously described (Dunny et al., 1979). To ensure that sufficient time had passed for the atmosphere within the Gas Pak jar to become anaerobic prior to culture filtrate preparation, anaerobically shaken cultures, along with still and aerated controls, were inoculated by diluting cultures grown to stationary phase by a factor of $10^{-6}$. Cultures were then grown for $\sim 16 \mathrm{~h}$ into early stationary phase. Bacterial colonies were grown anaerobically by incubating agar plates in a Gas Pak anaerobic jar.

Pheromone assays. The concentration of cAD 1, cPD1, and cAM373 in culture filtrates was determined by the microtiter assay method described previously (Dunny et al., 1979). Responder strains were DS16 (To- mich et al., 1979), 39-5 (Dunny et al., 1978), and FA373 (Clewell et al., 1985) for cAD1, cPD1, and cAM373 assays, respectively. The inhibitor iAD1 titer was determined as previously described (Ike et al., 1983). DS16 was used as responder in iAD1 assays.

$\beta$-Galactosidase assays. Still and aerated cultures were grown overnight as described above, diluted $1: 10$, and allowed to grow for 2 $h$ under the same conditions as the overnight inoculum. Induced cultures were exposed to $40 \mathrm{ng}$ of $\mathrm{cAD} 1 / \mathrm{ml} 30 \mathrm{~min}$ after dilution. Anaerobically shaken cultures were grown as described above and assayed along with similarly grown still and shaken cultures. Cells were harvested, toluenized, and assayed for $\beta$-galactosidase activity as previously described (Weaver and Clewell, 1988). Culture filtrates were prepared from supernatants of harvested cultures to ensure that culture con- 
TABLE 1

PLASMIDS $^{a}$

\begin{tabular}{|c|c|c|}
\hline Plasmid & Insert and relevant phenotype & Reference \\
\hline pAM2005A & NR5 insert; cAD1-inducible $\beta$-Gal production; agg ${ }^{-}, \mathbf{t f}^{-}$ & Weaver and Clewell, 1988 \\
\hline pAM2030 & A3 insert and deletion; constitutive agg, tf, cAD1 production & Clewell and Weaver, 1989 \\
\hline pAM2040 & B4 insert; constitutive agg and tf & Weaver and Clewell, 1988 \\
\hline pAM2040E & $\begin{array}{l}\text { B4 insert; pAD1 EcoRI B fragment mini-plasmid; growth } \\
\text { inhibition }\end{array}$ & Weaver and Clewell, 1988 \\
\hline pAM2045 & $\begin{array}{l}\text { NR5 and B4 inserts; constitutive } \beta \text {-galactosidase production } \\
\text { (but see text), cAD1 production }\end{array}$ & Weaver and Clewell, 1990 \\
\hline pAM2120 & A 120 insert; constitutive agg and tf & Weaver and Clewell, 1988 \\
\hline pAM2125 & NR5 and A 120 inserts; constitutive $\beta$-galactosidase production & Weaver and Clewell, 1990 \\
\hline pAM2140 & M14 insert; partial constitutive agg and tf, reduced response & Weaver and Clewell, 1988 \\
\hline $\mathrm{p} \wedge \mathrm{M} 2145$ & $\begin{array}{l}\text { NR5 and M14 inserts; partial constitutive } \beta \text {-galactosidase } \\
\text { production, reduced response }\end{array}$ & Weaver and Clewell, 1990 \\
\hline pAM2270 & PR27 insert and deletion; partial constitutive agg and tf & Weaver and Clewell, 1988 \\
\hline pAM2300 & B30 insert; constitutive agg and tf & Weaver and Clewcll, 1988 \\
\hline pDAK2010K & pAD1 "K-mini" derived from pAM2010 & This study \\
\hline pDAK2270E & PR27 insert; pAD1 EcoRI B fragment mini-plasmid & This study \\
\hline pDAK2270K & pADI "K-mini" derived from pAM 2270 & This study \\
\hline pDAK2300E & $\begin{array}{l}\text { B30 insert; pADI EcoRI B fragment mini-plasmid; growth } \\
\text { inhibition }\end{array}$ & This study \\
\hline pDAK2300K & pAD1 "K-mini" derived from pAM 2300 & This study \\
\hline
\end{tabular}

${ }^{a}$ All plasmids are pAD1 derivatives containing one, two, or a portion of a Tn917-lac insert. Double-insert plasmids pAM2125, pAM2145, and pAM2045 contain the inserts in opposite orientation. The plasmid contents of "K-minis" are shown in Fig. 2 and their construction is described under Materials and Methods. agg, aggregation in liquid medium; tf, conjugative transfer.

ditions had the expected effects on CAD1 production.

Mating experiments. To determine whether aeration affected constitutive transfer (conjugative transfer without prior exposure to cAD1) of various mutants, still, aerobic, and anaerobically shaken cultures were grown from $10^{-6}$ dilutions of a still culture. After $\sim 16 \mathrm{~h}$, cultures were diluted $10^{-3}$ and grown $\sim 6 \mathrm{~h}$ to late log phase. Cultures were then mated for $10 \mathrm{~min}$ in liquid medium without shaking as described previously (Ike and Clewell, 1984) with FA2-2 as recipient. Transconjugants were selected on N2GT agar plates supplemented with $25 \mu \mathrm{g}$ of rifampin and fusidic acid/ml and $10 \mu \mathrm{g}$ of erythromy$\operatorname{cin} / \mathrm{ml}$.

To observe the effect of aeration on pAM2040 transfer, it was essential to maintain the culture in log phase because adherence of cells to the side of the flask and culture settling in stationary phase increased the tendency of the cells to self-aggregate. For this reason, pAM2300 also was used to ensure that the effects of aeration were consistent in other traB mutants. OG1X (pAM 2300) did not self-aggregate when shaken.

DNA manipulations and construction of mini-plasmids. DNA was prepared by the CsCl-ethidium bromide equilibrium density gradient procedure previously described (Clewell et al., 1974). For rapid preparation and analysis of plasmid DNA, samples were prepared by the modified alkaline lysis procedure previously described (Weaver and Clewell, 1988). Plasmid constructs were analyzed by digestion with various restriction enzymes and separation of restriction fragments on $0.8 \%$ agarose gels. All restriction enzymes were obtained from Bethesda Research Laboratories, Inc. (Gaithersburg, MD) and reactions carried out under the conditions recommended.

Mini-plasmids were constructed basically as previously described (Weaver and Clewell, 1989). Briefly, plasmid DNA containing the 
TABLE 2

EFfects of Aeration on CADI Production and Expression of Conjugative Transfer Functions

\begin{tabular}{|c|c|c|c|c|c|c|c|}
\hline \multirow[b]{2}{*}{ Strain } & \multirow[b]{2}{*}{ Plasmid genotype ${ }^{d}$} & \multicolumn{2}{|c|}{ cAD1 titers $^{a}$} & \multicolumn{2}{|c|}{$\begin{array}{c}\beta \text {-Galactosidase } \\
\text { production }^{b}\end{array}$} & \multicolumn{2}{|c|}{ Conjugation frequency ${ }^{c}$} \\
\hline & & Still & Aerated & Still & Aerated & Still & Aerated \\
\hline OG1X & None & 256 & 16 & $-^{e}$ & - & - & - \\
\hline FA2-2 & None & 32 & 4 & - & - & - & - \\
\hline OG1X (pAM2030) & $\operatorname{traB} / C$ deletion & 64 & 4 & - & - & $1.2 \times 10^{-3}$ & $3.2 \times 10^{-4}$ \\
\hline OG1X (pAM2045) & $\operatorname{traB} / E(\mathrm{~B} 4 / \mathrm{NR} 5)$ & 32 & $<2$ & 64.9 & 0.9 & 一 & - \\
\hline OG1X (pAM2040) & $\operatorname{traB}(\mathrm{B} 4)$ & $<2-2^{f}$ & $<2$ & - & - & $2.4 \times 10^{-3}$ & $4.6 \times 10^{-6}$ \\
\hline OG1X (pAM2300) & $\operatorname{traB}(\mathrm{B} 30)$ & $<2$ & $<2$ & - & - & $1.1 \times 10^{-3}$ & $<7 \times 10^{7}$ \\
\hline OG1X (pAM2125) & $\operatorname{traA} / E(\mathrm{~A} 120 / \mathrm{NR} 5)$ & $<2$ & $<2$ & 71.1 & 63.2 & - & - \\
\hline OG1X (pAM2120) & $\operatorname{traA}(\mathrm{A} 120)$ & $<2$ & $<2$ & - & - & $4.0 \times 10^{-3}$ & $1.2 \times 10^{-2}$ \\
\hline OG1X (pAM2145) & $\mathrm{C} / \mathrm{E}(\mathrm{M} 14 / \mathrm{NR} 5)$ & $<2-2^{f}$ & $<2$ & 15.5 & 14.6 & - & - \\
\hline OG1X (pAM2140) & $\mathrm{C}(\mathrm{M} 14)$ & $<2$ & $<2$ & - & - & $3.2 \times 10^{-4}$ & $2.2 \times 10^{-4}$ \\
\hline OG1X (p^M2005^) & E (NR5) & $<2$ & $<2$ & 1.8 & 0.5 & - & -- \\
\hline
\end{tabular}

${ }^{a}$ cADl titer is defined as the highest dilution of culture filtrate still able to induce an aggregation response in a pAD1-containing stain, in this case DS16. Titers shown are representative of at least two experiments.

${ }^{b} \beta$-Galactosidase levels are representative of the level of expression of the cAD1-inducible E region fusion NR5. All other Tn917-lac fusions produce $\beta$-galactosidase at levels too low to be detected under the conditions used (Weaver and Clewell, 1990). Activities are expressed as Miller Units (Miller, 1972) and represent an average of at least three experiments.

${ }^{c}$ Conjugation frequencies were determined in 10-min matings as described under Materials and Methods. This represents constitutive expression of conjugative transfer functions. Frequencies are expressed as transconjugants per donor cell and are an average of at least two experiments.

${ }^{d}$ Genotypes represent the genes and/or regions of pAD1 derivatives containing Tn917-lac inserts or, in the case of pAM2030, the extent of a $\operatorname{Tn}_{n} 917$-lac-related deletion. Particular inserts are indicated in parentheses (see Fig. 1).

${ }^{e}$ - not applicable. $\beta$-Galactosidase activities are relevant only for plasmids containing $E$ region fusions. These plasmids are incapable of transfer due to loss of $\mathrm{E}$ region function and were not tested in conjugation experiments.

${ }^{f} \mathrm{OG} 1 \mathrm{X}$ strains containing pAM2040 and pAM2145 inconsistently produced low levels of cAD1.

insert of interest was digested with $K p n I$, which cuts once within the plasmid $E c o$ RI B fragment, just to the right of (clockwise from) the putative replication region, and once within Tn917-lac just outside the erm gene. The digested plasmid DNA then was ligated with T4 DNA ligase (Bethesda Research Laboratories, Inc.) and used to transform $E$. faecalis protoplasts to erythromycin resistance (Wirth et al., 1986). This resulted in the isolation of mini-plasmids consisting only of the pAD1 DNA located between the Tn917-lac insert and the plasmid KpnI site (see Fig. 1).

\section{RESULTS}

Effects of Aeration on E. faecalis Pheromone Production

As shown in Table 2, aeration resulted in significantly less CADI activity in culture fil- trates from OG1X and FA2-2 than in culture filtrates from still cultures. This effect was specific for CAD1, as no difference in CPD1 or cAM373 activities (the pheromones specific for $\mathrm{pPD} 1$ and pAM373, respectively) was observed under these conditions (data not shown). Culture filtrates from OG1X strains containing pAM2030 (traB/C deletion) and pAM2045 [E region (NR5) and traB (B4) inserts], two pADl derivatives previously shown to be defective in pheromone shutdown (see Fig. 1), also had significantly less cAD1 activity when prepared from aerated cultures. Aeration had no effect on IAD1 activities in $\mathrm{pAD} 1$-containing strains of OGIX or FA2-2.

To ensure that the decrease in cAD1 production was due to aeration and not to some other effect of shaking, culture filtrates were 
prepared from cultures grown in an anaerobic jar which was shaken vigorously (see Materials and Methods). cAD1 titers were 512 and 32 for OG1X and OG1X (pAM2045), respectively, identical to or slightly higher than activities observed in culture filtrates from still cultures.

The lower CADI titers observed in culture filtrates from aerated cultures werc not due to direct inactivation of CAD1 by aeration. Shaking of solutions of synthetic CAD1 or of culture filtrates prepared from still cultures did not result in a decrease in CAD1 titer. In addition, mixing of culture filtrates from aerated cultures with solutions of synthetic cAD1 or culture filtrates from still cultures did not result in a decrease in cAD1 activity beyond that expected due to dilution (data not shown). This indicated that aerated cultures did not excrete a product into the medium which specifically degraded CAD1.

\section{Effects of Aeration on Mutant Phenotypes: \\ Involvement of traB in $C A D 1$ Shutdown}

It was suggested previously (Weaver and Clewell, 1990) that the primary, and perhaps only, defect in pADI traB mutations is a failure to shut down CADl production properly. According to this theory, constitutive expression of mating functions in $t r a B$ mutants is due to a response to endogenously produced cAD1, which is undetectable by microtiter assay (perhaps because of the function of $E$ or $\mathrm{C}$ region gene products). If this is so, aeration of cells containing pAD1 traB mutations might be expected to reduce endogenous cAD1 levels below that required to induce the conjugative response; that is, aeration might be expected to alleviate the traB-related derepression of conjugative functions. Results presented below show, without exception, that aerobic incubation decreased, while anaerobic incubation increased, $t r a B$ related derepression of $\mathrm{pAD} 1$ conjugative functions, consistent with the postulated role of $t r a B$ in pheromone shutdown.

As shown in Table 2 , culture aeration effected both the expression of the NR5 E re- gion $l a c Z$ fusion and constitutive transfer in tra $B$ mutants. In pAM2045 the presence of the $\operatorname{Tn} 917-l a c$ insert $\mathrm{B} 4$ (traB) resulted in constitutive expression of the normally cAD1-inducible NR5 fusion in still cultures. Aeration resulted in $\sim 74$-fold less $\beta$-galactosidase expression from the fusion, coincident with the loss of detectable cAD1 activity in the culture filtrate. In contrast, aeration had little or no effect on the fusion alone (pAM2005A) or when present with $\operatorname{traA}$ A120 (pAM2125) or C region M14 (pAM 2145 ) inserts. Levels of $\beta$-galactosidase expression from pAM2045 were only slightly lower than those of still cultures when grown with shaking in an anaerobic jar. Enzyme activities averaged 90,11 , and $79 \mathrm{MU}$ in still, shaken (aerated), and anaerobically shaken cultures, respectively, indicating that aeration and not shaking itself was the primary cause of the observed difference. The reasons for the uniformly higher activities in this experiment, compared to those shown in Table 2 , is probably related to the different culture conditions used (see Materials and Methods). Expression of $\beta$-galactosidase in aerobically grown pAM2045-containing cells was inducible to levels comparable to those observed in induced pAM2005A-containing cells by exposure to CAD1 (data not shown).

Plasmids containing single Tn917-lac inserts within traB, such as pAM2040 (B4) and pAM2300 (B30), express conjugative transfer functions constitutively, as evidenced by transfer at high frequency in 10min matings-matings too short to allow induction by cADI produced by the recipient -but do not produce detectable cAD1. Nevertheless, aeration of OG1X (pAM2040) and OG1X (pAM2300) prior to mating resulted in a drop in constitutive mating frequency of more than 2 orders of magnitude (Table 2). OG1X (pAM2040) donor cultures shaken anaerobically transferred at a frequency of $\sim 1.2 \times 10^{-3}$, indicating again that aeration and not shaking itself is responsible for the observed effect. The effect also was not due to the selection of transfer-deficient mutants since aerated donor cells regrown in 


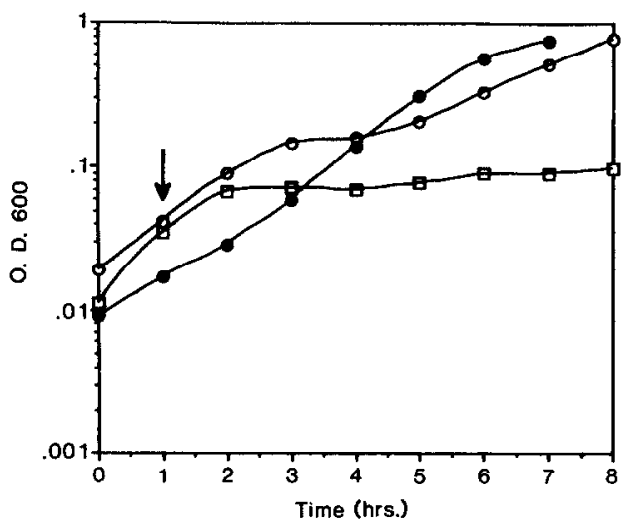

FIG. 2. Effect of aeration on the growth inhibition of OGIX (pDAK2300E). Cells were grown from single colony inocula to stationary phase in aerobic or still N2GT culture as described under Materials and Methods. These cultures were used to inoculate fresh medium at $2 \%$ (v/v). Symbols: - aerated; $O$, still; $\square$, still $+40 \mu \mathrm{g} / \mathrm{ml}$ cAD1. Synthetic cADl was added at the point marked with the arrow. Similar results were obtained with OGIX (pAM2040E).

still cultures regained their ability to transfer at high frequency in short matings (data not shown). Constitutive transfer of traA (pAM 2120 ) and $C$ region (pAM2140) mutants was not affected by aeration, indicating that constitutive transfer in these mutants is not due to a response to endogenously produced cAD1. Aeration only marginally affected constitutive transfer of the $\operatorname{traB} / \mathrm{C}$ region deletion mutant pAM2030, despite a signifcant decrease in CADI activity. However, the levels of $\mathrm{cADI}$ produced in aerated cultures containing this plasmid are still sufficient to induce conjugative functions.

It has been previously observed that pheromone induction of strains carrying any one of several pAD1 mini-plasmids results in a dramatic decrease in growth rate (Weaver and Clewell, 1989, 1990). This effect is believed to be due to pheromone-induced overexpression of a determinant located in the pAD1 replication region. pDAK2300E is a pAD1 mini-plasmid consisting of the pAD1 $E c o$ RI B fragment and the B30 (traB) insert. As shown in Fig. 2, exposure of OG1X (pDAK2300E) to exogenously added cAD1 resulted in a decrease in growth rate. Interest- ingly, after OG1X (pDAK2300E) cultures reached an optical density at $600 \mathrm{~nm}$ of $\sim 0.150$, the growth rate was decreased to approximately one-eighth of normal, even in the absence of added cAD1. It was postulated that growth inhibition resulted from the accumulation of endogenously produced cAD1. If this is so, aeration should eliminate the observed growth inhibition by reducing the level of CAD1 produced. As shown in Fig. 2 aeration had precisely that effect on the growth of OG1X (pDAK2300E).

Finally, aeration appears to affect the derepression of aggregation functions in colonies of strains containing pAD1 traB mutations. Indeed, it was the unique colony morphology of cells containing pAD 1 traB mutations that led us to consider the possibility that aeration might affect $\mathrm{CAD} 1$ production. Figure 3 shows the colony morphologies of OG1X strains containing wild-type pAD1, pAM $2120(\operatorname{tra} A)$, and pAM2300 (traB). OG1X (pAM2120) colonies were brighter and grainier than OG1X (pAD1) colonies and fractured when touched with a toothpick (Fig. $3 \mathrm{C}$ ). This is the typical "dry" colony morphology of traA mutants (Ike and Clewell, 1984). "Dry" colonies were also produced by OG1X (pAD1) when grown on plates containing cAD1 (Weaver and Clewell, 1988), indicating that colony dryness is related to expression of aggregation functions (not shown). Aerobically grown OG1X (pAM2300) colonies were dry in the center but were surrounded by a dull, watery ring (Fig. 3E), indicating that cells in the colony center were expressing aggregation functions while those on the edge were not. In contrast, anaerobically grown OG1X (pAM2300) colonies were uniformly dry (Fig. 3F) and indistinguishable from OG1X (pAM2120) colonies, which were unaffected by anaerobic growth (Figs. $3 \mathrm{C}$ and $\mathrm{D}$ ). While OG1X (pAD1) colonies displayed some increase in brightness when grown anaerobically, they did not fracture (Figs. 3A and B). If traB mutations result in a failure to properly shut down CAD1 activity, as postulated, colony dryness results from aggregation due to a response to endogenously 
produced cAD1. The increased aggregation under anaerobic conditions, conditions under which $\mathrm{CAD} 1$ production would be at its maximum, is consistent with this hypothesis. Aerobically grown colonies produce suffrcient CAD1 to induce aggregation functions only at the center of colonies, where increased cell density probably provides a more anaerobic environment.

Production of $\beta$-galactosidase in OG1X (pAM2045) colonies on X-gal-containing plates mirrored the expression of aggregation functions in OG1X (pAM2040) colonies. Thus, OG1X (pAM2045) colonies had blue centers and white outer edges. In addition, streaks of OG1X (pAM2045) made perpendicularly across horizontal slices made in the agar produced blue pigment predominantly in and around the slice, where conditions presumably were sufficiently anaerobic to increase cAD1 levels. OG1X (pAM2125) and OG1X (pAM2005A) colonies produced uniformly blue and white streaks, respectively.

\section{Identification of pAD1-Encoded Genetic Determinants Involved in Pheromone Shutdown}

The results presented above indicated that the product of the $\operatorname{tr} B$ gene was required for normal pheromone shutdown, even though transposon insertion mutants in this gene did not consistently produce levels of cADI detectable in culture filtrates. To determine whether any other plasmid-encoded determinants were essential for pheromone shutdown, mini-plasmids containing various portions of the pheromone response control region were constructed (Fig. 1). Culture filtrates were prepared from still cultures of OG1X strains containing each of these plasmids. In the absence of any intact phero- mone response regulatory genes (pDAK $2300 \mathrm{~K}$ ), cAD1 activity was identical to that observed in plasmid-free strains (cAD1 titer $=256$ ). The presence of $\operatorname{tra} B$ alone (pDAK2270K) resulted in a four- to eightfold drop in cAD1 activity (cAD1 titer $=64$ ), indicating that the $t r a B$ product is involved in but not sufficient for pheromone shutdown. The addition of the $C$ region function to $\operatorname{traB}$ (pDAK2010K) resulted in undetectable cADI activity, indicating that the products of both of these regions contribute to pheromone shutdown. However, $\mathrm{C}$ region function was not essential for pheromone shutdown, as mini-plasmids constructed with a deletion of the C region (pDAK2270E) did not allow production of detectable cAD1 activity. Differences in cADI activities were not due to differences in stability or copy number of the plasmid constructs, as all plasmids were stable over the period of time used to prepare culture filtrates and all had normal copy numbers ( 2 to 4 copies per chromosomal equivalent). Therefore, it would appear that the product of the $\operatorname{tra} B$ gene required the cooperation of either the $C$ region product or some other product encoded within the pheromone response regulatory region to prevent detection of cAD1. These results are consistent with mutagenesis experiments which revealed that mutations disrupting $\operatorname{tr} a B$ and either the $\mathrm{E}$ or $\mathrm{C}$ region produced cAD1, while those disrupting $\operatorname{tr} a B$ alone did not produce detectable CADI but had a phenotype suggestive of a response to endogenous pheromone (Weaver and Clewell, 1990). A subset of mutations within the C region (e.g., pAM2140) also showed some evidence of constitutive expression of conjugation functions (Weaver and Clewell, 1988), but the fact that aeration did not affect the expression of these functions (e.g., constitu-

FIG. 3. Colony morphology of OGIX (pAD1) (wild-type), OGIX (pAM2120) (traA), and OG1X (pAM2300) $(\mathrm{tr} a B)$ grown under aerobic and anaerobic conditions. Cells were streaked from frozen stocks onto N2GT medium for isolation. Plates were incubated at $37^{\circ} \mathrm{C}$ for $18-24 \mathrm{~h}$. Photographs were taken with an Olympus dissecting microscope at $40 \times$ magnification. Plates were illuminated from underneath with reflected light. Frames: (A and B) OGIX (pAD1) aerobic and anaerobic, respectively; (C and D), OG1X (pAM2120) aerobic and anaerobic, respectively; (E and F) OG1X (pAM2300) aerobic and anaerobic, respectively. 

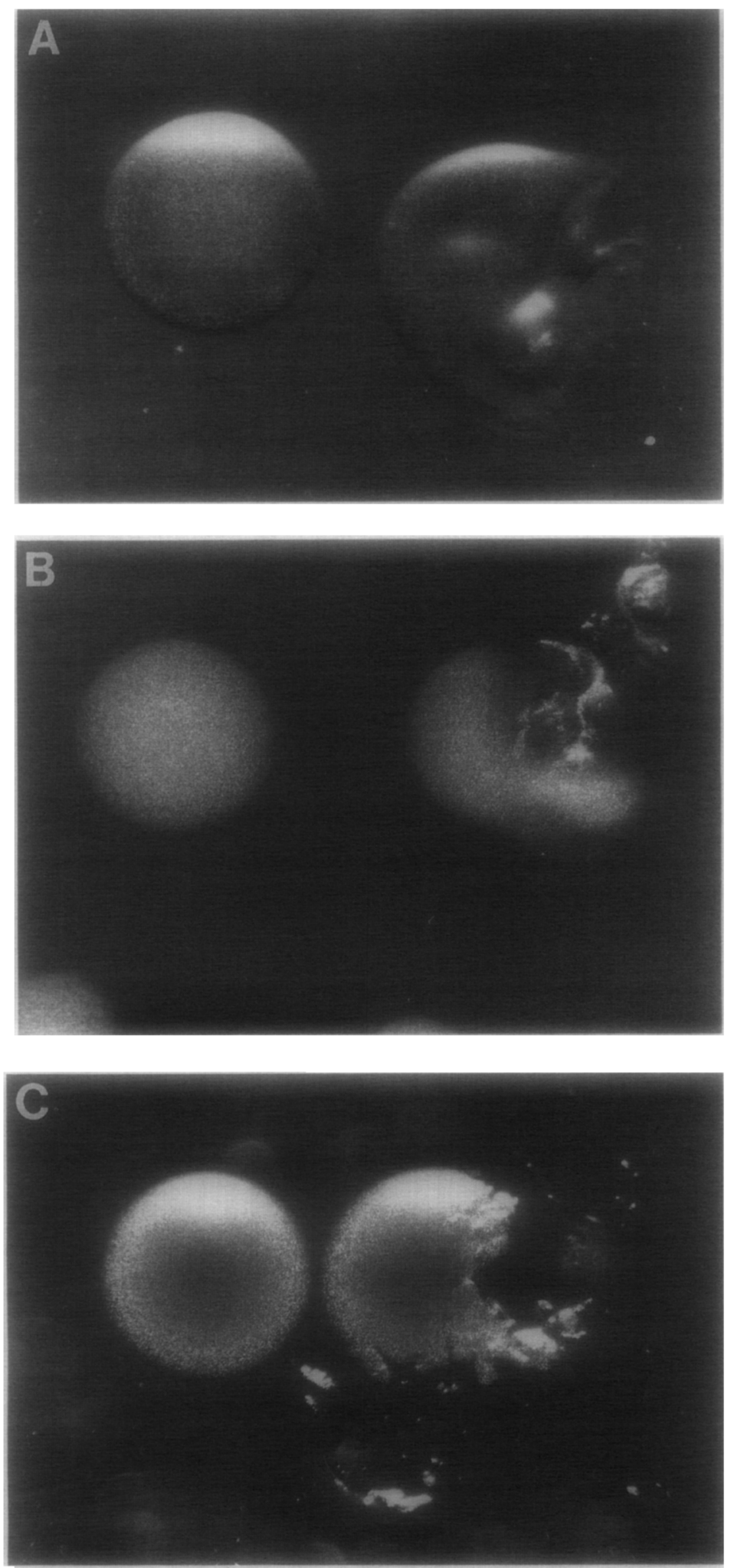

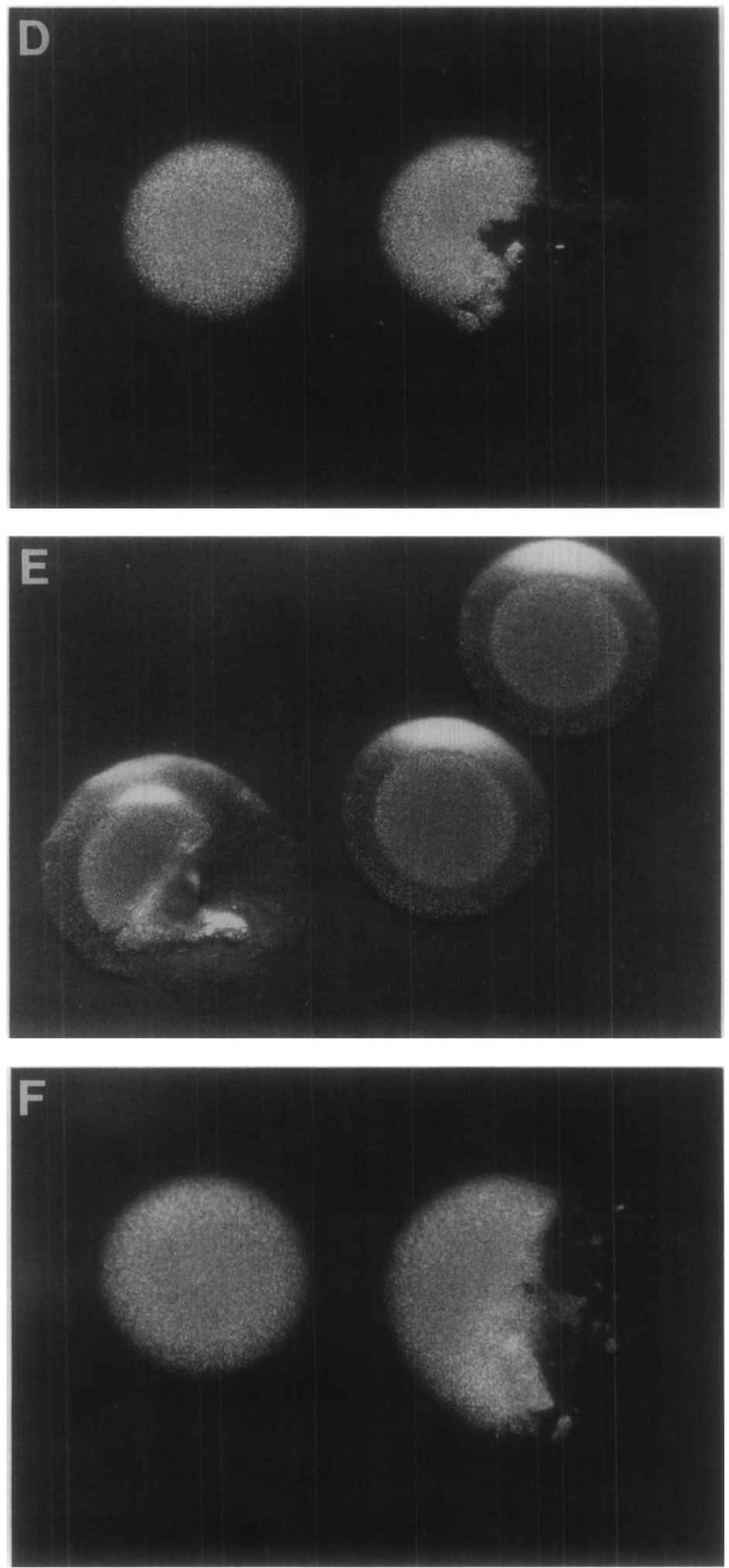

FIG. 3-Continued 
tive transfer, see Table 2) indicates that C-region-related derepression is not due to production of endogenous CADI.

\section{DISCUSSION}

Pheromone production in E. faecalis is regulated by plasmid-encoded determinants which specifically shut down the pheromone to which the plasmid responds. We report the added involvement of an environmental factor, namely culture aeration, in the regulation of $\mathrm{CAD}$, the pheromone specific for pAD1. Aeration of plasmid-free strains of $E$. faecalis or strains containing pAD1 derivatives defective in pheromone shutdown consistently resulted in $\sim 16$-fold less CADl activity. Aeration had no effect on the activities of two unrelated pheromones, cPD1 and cAM373. Because little is known about the mechanisms of production and excretion of pheromone, it is difficult to speculate on the mechanisms of, or reasons for, the specific effect of aeration on CAD 1 activity. However, since $E$. faecalis is a normal inhabitant of the human intestinal tract, where anaerobiosis is a common condition, this form of regulation may provide some advantage. It is noteworthy that the hemolysin-bacteriocin determinant of the related family of plasmids that respond to $\mathrm{CAD} 1$ has been implicated in virulence (Ike et al., 1984, 1987).

Previous results indicated that $\operatorname{traB}, \mathrm{C}$, and $E$ region determinants were involved in cAD I shutdown, but that single mutations in any of these determinants failed to allow excretion of measurable amounts of cAD1. However, the constitutive expression of conjugative transfer functions in $\operatorname{traB}$ mutants suggested that such plasmids may be responding to $\mathrm{CAD} 1$ produced endogenously at levels too low to detect by microtiter assay (Weaver and Clewell, 1990). If this hypothesis is correct, decreasing endogenous $\mathrm{CAD} 1$ activity by aeration would be expected to decrease or eliminate the $t r a B$-related derepression of pAD1 transfer functions. Without exception, aeration had the expected effect on the $\operatorname{tra} B$ phenotype. Thus, (i) aeration vir- tually eliminated the $t r a B$-related derepression of the $E$ region lac $Z$ fusion of pAM2045, (ii) aeration decreased the constitutive transfer frequencies of the $t r a B$ mutant plasmids pAM2300 and pAM2040, and (iii) aeration eliminated the observed growth inhibition of cells containing the mini-plasmids pAM2300E and pAM2040E. Even the unique "ringed" colony morphology of cells containing pAD1-linked traB mutations can be explained as a combination of the failure of such cells to properly shut down cAD1 activity and the effect of aeration on CAD1 activity. Thus, cclls on colony edges of acrobically grown colonies do not produce enough cADl to induce pAD1-encoded aggregation functions. As colony centers become more densely packed with cells, the environment becomes sufficiently anaerobic to increase cAD1 production. traB mutants fail to properly control the elevated cAD1 activity and aggregation ensues. Such effects of colony depth on oxygen penetration have been reported previously for Bacillus cereus, Staphylococcus albus, and Escherichia coli (Peters et al., 1987). Anaerobic incubation resulted in uniformly "dry" colonies presumably because CAD1 was produced at substantial levels in all portions of the colony. Cellular differentiation occurring within colonies of $E$. coli has been reported previously (Shapiro, 1987).

The universal effect of aeration on $\operatorname{traB}$-related phenotypes indicates that the $\operatorname{tr} B$ gene product is essential for CAD1 shutdown and has its primary, and perhaps sole, effect on the regulation of $\mathrm{CAD} 1$ activity. In contrast, aeration had no effect on traA- or C-regionrelated derepression of transfer functions, indicating that constitutive expression of conjugative functions in these mutants is not due to response to endogenous pheromone. In the case of $\operatorname{traA}$, this is consistent with that gene product's postulated role as a repressor of $E$ region transcription. The reason for the slight derepression of transfer functions observed in some $\mathrm{C}$ region mutants, like pAM2140 (Weaver and Clewell, 1988), remains a mystery. The failure of aeration to 
decrease $\beta$-galactosidase production in pAM 2145-containing cells indicates that C-region-related derepression is not due to the small amounts of CAD1 produced (Weaver and Clewell, 1990). Therefore, the derepression of mating functions observed in $\mathrm{C}$ region mutants apparently is unrelated to its role in pheromone shutdown.

Results with mini-plasmids containing various portions of the $\mathrm{pAD} 1$ pheromone response regulatory region revealed that $\operatorname{tr} a B$ alone was not sufficient for CAD1 shutdown but that one or more other gene products encoded within that region also were required. While $\operatorname{tr} B$ and the $C$ region were sufficient to encode pheromone shutdown functions, the $\mathrm{C}$ region was not essential and could be substituted for by another gene located on the pAD1 EcoRI B fragment. The production of detectable levels of $\mathrm{CAD} 1$ by cells containing pAM2045 had led us to postulate that an inducible $\mathrm{E}$ region product may serve as a feedback inhibitor of the pheromone response, perhaps by inactivating CAD1 (Weaver and Clewell, 1990). Alternatively, iAD1, which also is known to be encoded within the pheromone response regulatory region (Weaver and Clewell, 1989; Clewell et al., manuscript in preparation), may interfere with detection of cAD1.

In conclusion, we report the specific effect of aeration on CAD1 activity and the exploitation of this phenomenon to confirm the importance of the $t r a B$ gene product in CAD1 shutdown. In addition, through the construction of various mini-plasmids, we have shown that $\operatorname{traB}$ is not sufficient for pheromone shutdown, but requires functions encoded within the $C$ region or elsewhere within the $\mathrm{pAD} 1$ pheromone response regulatory region.

\section{ACKNOWLEDGMENTS}

We thank L. Pontius, M. Sulavik, F. An, S. Flanagan, L. Washburn, and R. Duman for helpful discussion and technical assistance. We also thank Dr. Harry Settles and the University of South Dakota Medical Audio-visual Services for their assistance in colony photography and the patient cooperation of the secretarial staff in prepar- ing and revising the manuscript. This study was supported by Public Health Service Grants GM33956 and Al 10318 from the National Institutes of Health and by a grant from the University of South Dakota/Parsons Medical School Research Fund.

\section{REFERENCES}

Clewell, D. B., AN, F. Y., White, B. A., AND Gawron-Burke, C. (1985). Sex pheromones and plasmid transfer in Streptococcus faecalis: A pheromone, cAM373, which is also excreted by Staphylococcus aureus. In "Plasmids in Bacteria" (D. R. Helinski, S. N. Cohen, D. B. Clewell, D. A. Jackson, and A. Hollaender, Eds.). Plenum, New York.

Clewell, D. B., Tomich, P. K., GaWron-Burke, M. C., Franke, A. E., Yagl, Y., AND AN, F. Y. (1982). Mapping of Streptococcus faecalis plasmids pAD1 and pAD2 and studies relating to the transposition of Tn917. J. Bacteriol. 152, 1220-1230.

Clewell, D. B., AND Weaver, K. E. (1989). Sex pheromones and plasmid transfer in Enterococcus faecalis. Plasmid 21, 175-184.

Clewell, D. B., Yagi, Y., DunNY, G. M., AND SCHulTZ, S. K. (1974). Characterization of three plasmid deoxyribonucleic acid molecules in a strain of Streptococcus faecalis: Identification of a plasmid determining erythromycin resistance. J. Bacteriol. 117 , 283-289.

Dunny, G. M., Brown, B. L., AND Clewell, D. B. (1978). Induced cell aggregation and mating in Streptococcus faecalis: Evidence for a bacterial sex pheromone. Proc. Natl. Acad. Sci. USA 75, 3479-3483.

DunNy, G. M., Craig, R. A., Carron, R. L., AND CleWELL, D. B. (1979). Plasmid transfer in Streptococcus faecalis: Production of multiple sex pheromones by recipients. Plasmid 2, 454-465.

EHRENFELD, E. E., AND ClEWELL, D. B. (1987). Transfer functions of the Streptococcus faecalis plasmid pAD1: Organization of plasmid DNA encoding response to sex pheromone. J. Bacteriol. 169, 3473-3481.

IKE, Y., AND CLEWELL, D. B. (1984). Genetic analysis of the pAD1 pheromone response in Streptococcus faecalis using $\operatorname{Tn} 917$ as an insertional mutagen. J. Bacteriol. 158, 777-783.

IKe, Y., Craig, R. C., White, B. A., YaGi, Y., AND Clewell, D. B. (1983). Modification of Streptococcus faecalis sex pheromone after acquisition of plasmid DNA. Proc. Natl. Acad. Sci. USA 80, 5369-5373.

IKe, Y., Hashimoto, H., AND Clewell, D. B. (1984). Hemolysin of Streptococcus faecalis subspecies zymogenes contributes to virulence in mice. Infect. Immun. 45, 528-530.

IKE, Y., Hashimoto, H., AND Clewell, D. B. (1987). High incidence of hemolysin production by Enterococcus (Streptococcus) faecalis strains associated with human parenteral infections. J. Clin. Microbiol. 25, 1524-1528.

Miller, J. H. (1972). “Experiments in Molecular Genet- 
ics." Cold Spring Harbor Laboratory, Cold Spring Harbor, NY.

Peters, A. C., Wimpenny, J. W. T., AND Coombs, J. P. (1987). Oxygen profiles in, and in the agar beneath, colonies of Bacillus cereus, Staphylococcus albus and Escherichia coli. J. Gen. Microbiol. 133, 1257-1263.

SHAPIRO, J. A. (1987). Organization of developing Escherchia coli colonies viewed by scanning electron microscopy. J. Bacteriol. 169, 142-156.

Tomich, P. K., AN, F. Y., Damle, S. P., ANd Clewell, D. B. (1979). Plasmid-related transmissibility and multiple drug resistance in Streptococcus faecalis subspecies zymogenes strain DS16. Antimicrob. Agents Chemother. 15, 828-830.

WEAVER, K. E., AND CleWell, D. B. (1988). Regulation of the pADI sex pheromone response in Enterococcus faecalis: Construction and characterization of lac $Z$ transcriptional fusions in a key control region of the plasmid. J. Bacteriol. 170, 4343-4352.

WEAVER, K. E., AND Clewell, D. B. (1989). Construction of Enterococcus faecalis pAD1 mini-plasmids: Identification of a minimal pheromone response regulatory region and evaluation of a novel pheromone-dependent growth inhibition. Plasmid 22, 106-1 19.

WEAVER, K. E., AND Clewell, D. B. (1990). Regulation of the pADI sex pheromone response in Enterococcus faecalis: Effects of host strain and $\operatorname{traA}, \operatorname{traB}$, and $\mathrm{C}$ region mutants on expression of an $\mathrm{E}$ region pheromone-inducible lacZ fusion. J. Bacteriol. 172, 26332641.

WiRTh, R., AN, F. Y., AND Clewell, D. B. (1986). Highly efficient protoplast transformation system for Streptococcus faecalis and a new Escherichia coli-S. faecalis shuttle vector. .J. Bacteriol. 165, 831-836. 